\title{
Innovative use of tourniquet in the management of an advanced abdominal pregnancy to achieve an unusually normal postoperative outcome: a case report
}

\author{
Emmanuel C. Inyang Etoh ${ }^{1 *}$, Iniobong E. Ettete ${ }^{1}$, Christian A. Adeneye ${ }^{2}$, \\ Imaobong O. Inwang ${ }^{2}$, Augustine V. Umoh'
}

\author{
${ }^{1}$ Department of Obstetrics and Gynecology, University of Uyo, Nigeria \\ ${ }^{2}$ Department of Obstetrics and Gynecology, University of Uyo Teaching Hospital, Nigeria
}

Received: 27 December 2019

Revised: 30 January 2020

Accepted: 05 February 2020

\author{
*Correspondence: \\ Dr. Emmanuel C. Inyang-Etoh, \\ E-mail: emmacol2000@yahoo.com
}

Copyright: () the author(s), publisher and licensee Medip Academy. This is an open-access article distributed under the terms of the Creative Commons Attribution Non-Commercial License, which permits unrestricted non-commercial use, distribution, and reproduction in any medium, provided the original work is properly cited.

\begin{abstract}
Mrs. UVG was an un-booked G3P1+1 petty trader, who presented with an obstetric ultrasound scan report, with an incidental diagnosis of abdominal pregnancy at 32 weeks of gestation with the placenta attached to the fundus of the uterus. Her admission packed cell volume was $24 \%$. She had pre-operative preparation and 2 units of compatible blood were transfused to correct the anemia. Four additional units of compatible blood were made available before she was scheduled for an exploratory laparotomy at 33 weeks of gestation. A grossly normal male infant weighing 2.2 $\mathrm{kg}$ was delivered from the peritoneal cavity with Apgar scores of 2 at 1 minute and the same at 5 minutes. The placenta which was attached to the fundus of the uterus was removed manually completely after a tourniquet had been applied distal to the point of separation. Intra-operative blood loss was $1000 \mathrm{ml}$. The infant died 1 hour after delivery due to respiratory failure. Autopsy report revealed massive intracerebral hemorrhage and pulmonary hypoplasia. The post-operative period was uneventful and the decline in serum assay of $\beta$-human chorionic gonadotrophin postpartum was normal. She was discharged home on the $8^{\text {th }}$ post-operative day and seen at the postnatal clinic twice at weekly intervals with normal serum assay of $\beta$-human chorionic gonadotrophin. Her 6 weeks postnatal visit was also uneventful.
\end{abstract}

Keywords: Abdominal pregnancy, Complete removal of placenta, Minimal hemorrhage, Tourniquet application, Uneventful postoperative period

\section{INTRODUCTION}

Abdominal pregnancy is a rare type of extra-uterine pregnancy, accounting for $1.0-1.5 \%$ of all such extrauterine pregnancies. ${ }^{1,2}$ The incidence of abdominal pregnancy is however commoner in the tropics due to the prevalence of sexually transmitted infections and pelvic septic morbidities. ${ }^{3,4}$ The recommended approach to the management of abdominal pregnancy is to terminate the pregnancy in the safest way possible once a diagnosis is made. ${ }^{5}$ However, due to underutilization of antenatal care and inadequacy of maternity care resources in developing countries, some mothers present late with advanced abdominal pregnancies, with increased risks of complications in pregnancy and during delivery. ${ }^{4,6}$

The high maternal mortality and perinatal morbidity and mortality attributed to abdominal pregnancy result largely from failure to diagnosis the condition early, the ectopic location of the fetus, as well as difficulties experienced in 
the course of manually removing the placenta. ${ }^{6,7}$ Maternal mortality from abdominal pregnancy is estimated at 5 per 1000 cases, 7 times higher than mortality from other ectopic pregnancies. ${ }^{6,7}$ Maternal mortality is often attributed to uncontrollable intraoperative haemorrhage. ${ }^{7,8}$ Due to the rarity of the condition and the difficulty experienced in manually removing a placenta that may be attached to a vital abdominal organ, experience can only be pooled through case reports.

This case report was a viable advanced abdominal pregnancy that was an incidental sonographic diagnosis with the placenta attached to the fundus of the uterus. It is reported to highlight the innovative technique of applying a tourniquet distal to the point of separation of the placenta to achieve complete placental removal, moderate intraoperative blood loss and a normal postoperative period. It is envisaged that this report may be of help to medical personnel who may encounter a similar scenario in their practice.

\section{CASE REPORT}

Mrs. UVG was an un-booked gravida 3, para 1+1 (induced abortion) petty trader, who presented at the antenatal clinic of the University of Uyo Teaching Hospital, Uyo at a gestational age of 32 weeks +4 days on account of an incidental finding of a live abdominal gestation following an ultrasound scan she did at a private diagnostic center.

The index pregnancy was conceived spontaneously and she did not obtain any form of antenatal care prior to presentation.

However, apart from the fact that she had an episode of lower abdominal pain that lasted for 3 days at the gestational age of 6 weeks, the course of the antenatal period was uneventful. There was no history of threatened abortion or antepartum hemorrhage. There was also no history of dizziness and fainting attacks.

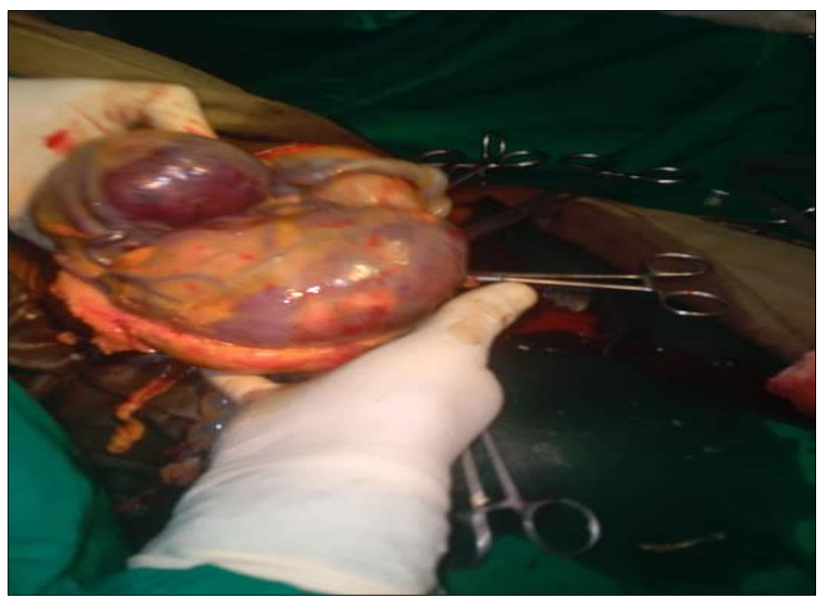

Figure 1: The hourglass - remodeled placenta before manual removal.

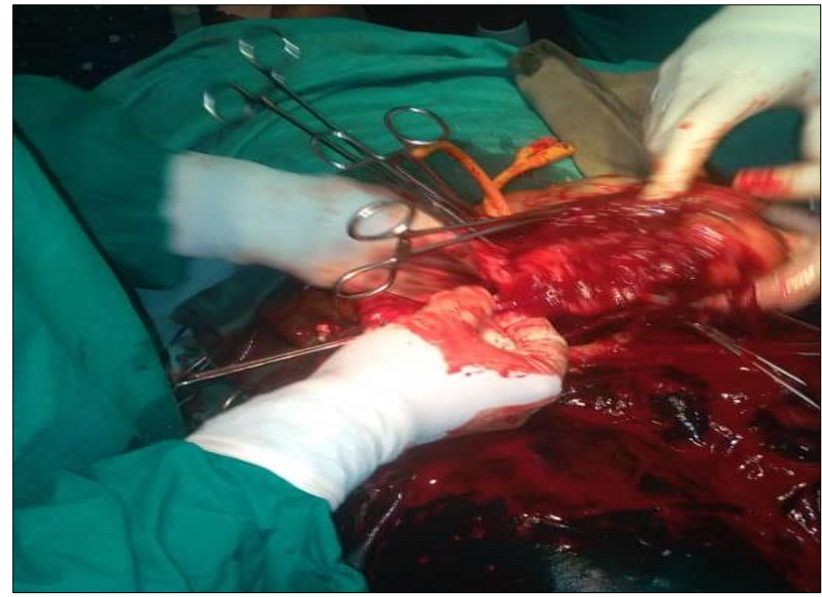

Figure 2: Application of tourniquet and manual removal of placenta.

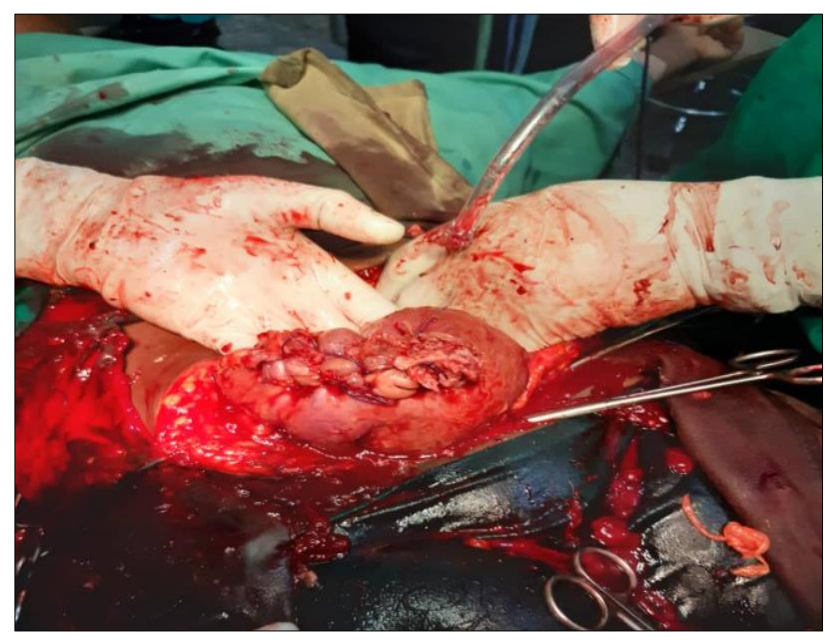

Figure 3: Placental bed at end of surgery.

At presentation, her vital signs were within normal limits. Abdominal examination revealed a symphysio-fundal height of $32 \mathrm{~cm}$, easily palpable fetal parts and fetal heart rate of 132 beats per minute. Her admission hematocrit was $24 \%$ and her urinalysis result was normal. She had a repeat obstetric ultrasound scan in the study center, which confirmed a live abdominal pregnancy at a gestational age of 32 weeks and 5 days. Her serum electrolytes/urea/creatinine were within normal limits and liver function test was normal.

Her vital signs were assessed every 2 hours and they remained stable. She was transfused with 2 units of settled blood cells and the post-transfusion hematocrit was $31 \%$. The general surgical and neonatology teams were invited to review the patient and contribute to her clinical management. On the $6^{\text {th }}$ day on admission at 33 weeks +3 days, she complained of abdominal discomfort, which was associated with fetal movements. Antepartum corticosteroids were administered intramuscularly to her with the hope of promoting fetal lung maturation. 
She had an exploratory laparotomy at a gestational age of 33 weeks +4 days with the general surgeon and neonatologist in attendance. Findings at laparotomy was a viable fetus lying freely in the peritoneal cavity. The amniotic sac had ruptured. The uterine size was comparable to a 14 weeks size of pregnancy. The placenta which was remodeled to adopt an hourglass shape was attached to the fundus of the uterus (Figure 1). The right fallopian tube was fibrotic and adherent to the body of the uterus. Both ovaries and the left fallopian tube were grossly normal.

After delivery of the fetus from the peritoneal cavity taking care not to put traction on the placental bed, the umbilical cord was doubly clamped and severed in between the clamps. A Foley catheter, size $16 \mathrm{~F}$ was applied as a tourniquet between the base of the placenta and the fundus of the uterus (Figure 2). The bulk of the placenta made up the upper bulb of the hourglass shape and was manually separated at the point of the relatively narrow neck. Residual placental tissue were removed manually from the lower bulb of the hourglass shape of the placenta. The lower bulb measure about $2 \mathrm{~cm}$ in height. The covering fetal membranes were stitched to overlie the raw surface of the placental bed. Series of figure 8 stitches were inserted at bleeding points to secure hemostasis. On removal of the tourniquet, there was no bleeding at the site of attachment of the placenta (Figure 3). Peritoneal lavage was performed and an intraperitoneal drain was inserted and the abdominal cavity closed in layers. Estimated blood loss was 1000 milliliters. She was transfused 2 units of whole blood intraoperatively. Informed consent was obtained from the patient to report this case; approval was equally received from the institutional Research Ethics Committee.

The infant had Apgar scores of 2 over 10 at 1 minute and the same at 5 minutes. Resuscitative measures did not improve the state of health of the infant who died 1 hour postpartum. Histology report of the placenta was normal but autopsy report of the infant revealed massive intracerebral hemorrhage and hypoplastic lungs with widespread pneumonitis. The mother had an uneventful post-operative period. The postoperative haematocrit was $28 \%$. Her initial postpartum serum assay of $\beta-\mathrm{HCG}$ was $211.3 \mathrm{miu} / \mu \mathrm{l}$ on the $2^{\text {nd }}$ postoperative day. Serum assay of $\beta$-HCG on the 7 th postoperative day was undetectable. She was discharged on the 8 th postoperative day, while the serial serum assay of $\beta-H C G$ on the $14^{\text {th }}$ and $21^{\text {st }}$ postoperative days remained undetectable during her initial weekly follow up visits. She was seen at 6 weeks postpartum at the postnatal clinic and her condition was normal.

\section{DISCUSSION}

The rarity of abdominal pregnancy, the risk of perinatal morbidity and mortality, as well as the challenges of managing primary hemorrhage that often follow manual removal of a placenta that might be attached to a delicate abdominal organ make a case for the continued need for case reports of every abdominal pregnancy encountered in practice. Such reports provide avenues for sharing of experiences gained. This case reported was sonographically diagnosed incidentally at advanced stage of pregnancy, but the diagnosis did not say whether the pregnancy was primarily abdominal or not.

Findings at laparotomy suggested that the pregnancy might have resulted secondarily from right tubal abortion, because the right fallopian tube showed signs of inflammatory reaction. In addition, the placenta was attached to the fundus of the uterus. This is in agreement with Studdiford's criteria, which states that for a primary abdominal pregnancy, the ovaries and tubes must be clinically normal, without any utero-peritoneal fistula and the pregnancy must be exclusively associated with the peritoneal surface. ${ }^{9-12}$ The fetus was delivered free in the abdominal cavity with very low Apgar scores of 2 over 10 because the amniotic sac had ruptured, exposing the fetus to pulmonary hypoplasia. In contrast, in cases where intra-abdominal fetuses are delivered with good Apgar scores, such fetuses are often retained in an intact amniotic sac at the time of delivery. $7,9,11,13$

Even though the infant died shortly after birth in this case reported, there were no pressure deformities, apparently because the infant was relatively small when compared to the space available to it in the mother's abdominal cavity. Much variation in the management of abdominal pregnancies as reported lies in how the placenta is handled following delivery of the fetus; while some obstetricians err on the side of caution and retain the placenta, others dare to remove the placenta with sometimes dire consequences. ${ }^{11-13}$

This is a case where a Foley catheter was used innovatively as a tourniquet to significantly reduce primary hemorrhage from the placental bed, while the placenta was manually removed completely. Similar cases where a tourniquet was not used in the way reported here, resulted in excessive loss of blood, in excess of 2000 milliliters. . $^{4,11}$ Where hemorrhage was that much, several units of blood were transfused. On the other hand, when the placenta is retained, the postoperative period could be turbulent, and there is often the need for methotrexate administration to induce autolysis of the placenta. ${ }^{4,7}$

With the innovative technique reported in handling placental removal, blood loss was minimal, transfusion was modest, the postoperative period was normal and there was no need for methotrexate therapy to induce autolysis of the retained placenta. ${ }^{5,10,13}$ These are benefits that could be exploited by other obstetricians confronted with a similar scenario. Prolonged follow-up of the mother in this case report was avoided, save for two initial weekly visits needed to confirm complete return of $\beta$-HCG to normal. Some cases report follow-up visits, sometimes as long as 6 months, especially when the 
placenta was retained. ${ }^{11-13}$ Such prolonged follow up could be avoided in the overall interest of the patients, the health personnel and the economy of the health facility.

\section{CONCLUSION}

In conclusion, we report the case of an un-booked live advanced abdominal pregnancy diagnosed at the gestational age of 32 weeks, which was discovered through a self-prescribed obstetric ultrasonography. There was mild anemia in pregnancy, which was corrected. The mother had an exploratory laparotomy with innovative application of a tourniquet distal to the placental attachment to the uterine fundus to attain minimal hemorrhage from the placental bed and complete removal of the placenta. The postoperative period was uneventful, even though the infant died one hour after birth due to respiratory failure.

Funding: No funding sources

Conflict of interest: None declared

Ethical approval: Not required

\section{REFERENCES}

1. Atrash HK, Friede A, Hogue CJR. Abdominal pregnancy in the United States: frequency and maternal mortality. Obstet Gynecol. 1987;69(3,1):333-7.

2. Gibbs RS. Incidence of abdominal pregnancy. In: Gibba RS. (Ed.) Danforths Obstetrics and Gynaecology. Lippincott Willcame and Wilkins, Philadelphia; 2008:84.

3. Gabkika BM, Ignome T, Diarra D. Abdominal pregnancy with a live fetus at term at the South N'djamena district hospital. A case study. Sudan J Med Sci. 2015;10(2):77-80.

4. Mutarambirwa HD, Kenfack B, Fongon JT. Term abdominal pregnancy revealed by amnioperitoneum in rural area. Case Rep Obstet Hynecol. 2017;4096783:1-7.
5. Isah AY, Ahmed Y, Nwobodo EI, Ekele BA. Abdominal pregnancy with a full-term live fetus: case report. Ann African Med. 2008;4(7):198-9.

6. Hamdan M, Rousseau G, Wagner J. Abdominal pregnancy. An autochtonous case with full term living normal infant. $\mathbf{J}$ de Chirurgie. 1991;128(12):544-7.

7. Abdullahi HM, Yakasai IA, Zakari M, Shuaibu DS. Late presentation of advanced abdominal pregnancy with a live baby: a case report and literature review. Nigerian J Basic Clin Sci. 2013;1(10):25-8.

8. Ejikeme BN, Umeora OUJ, Mbazor JO, Egwuatu VE. Abdominal pregnancy presenting with antepartum haemorhage: a case report. Sahel Med J. 2006;2(9):69-70.

9. Huang K, Song L, Wang L, Gao Z, Meng Y, Lu Y. Advanced abdominal pregnancy: an increasingly challenging clinical concern for obstetricians. Int $\mathrm{J}$ Clin Exp Pathol. 2014;7(9):5461-72.

10. Agarwal N, Odejinmi F. Early abdominal ectopic pregnancy: challenges, update and review of current management. The Obstet Gynaecol. 2014;16:193-8.

11. Mengistu Z, Getachew A, Adefris M, Musinguzi E. Term abdominal pregnancy: a case report. J Med Case Rep. 2015;9:168.

12. Osanyim GE, Okunade KS, Oye-Adeniran BA. A case report of a successfully managed advanced abdominal pregnancy with favorable feto maternal outcomes. TJOG. 2017;3(34):240-2.

13. Baffoe P, Fofie C, Gandau BN. Term abdominal pregnancy with healthy newborn: a case report. Ghana Med J. 2011;2(46):81-3.

Cite this article as: Inyang-Etoh EC, Ettete IE, Adeneye CA, Inwang IO, Umoh AV. Innovative use of tourniquet in the management of an advanced abdominal pregnancy to achieve an unusually normal postoperative outcome: a case report. Int J Reprod Contracept Obstet Gynecol 2020;9:1292-5. 